\title{
Incertidumbre del precio internacional del petróleo y rendimientos accionarios en México a través de un SVAR-MGARCH
}

\author{
Uncertainty of the international oil price and stock returns in Mexico through \\ an SVAR-MGARCH
}

\section{Domingo Rodríguez Benavides ${ }^{1 *}$, Miguel Ángel Martínez García² y Luis Fernando Hoyos Reyes ${ }^{1}$}

\author{
${ }^{1}$ Universidad Nacional Autónoma de México, México \\ ${ }^{2}$ Universidad Autónoma Metropolitana, México
}

Recibido el 5 de diciembre de 2018; aceptado el 26 de marzo de 2019

Disponible en Internet el:30 de abril de 2019

\section{Resumen}

En este trabajo examinamos el impacto de la incertidumbre del precio del petróleo en los rendimientos del mercado accionario de México. La incertidumbre del precio internacional del petróleo es aproximada mediante la desviación estándar condicional del pronóstico de error de un paso adelante de la variación en el precio del petróleo. Con tal fin estimamos un SVAR-MGARCH en media con datos mensuales de los rendimientos del precio internacional del petróleo y del índice de precios y cotizaciones de la Bolsa Mexicana de Valores, ambos en términos reales de enero de 1975 a septiembre de 2018, cuya principal ventaja es que permite la estimación simultánea tanto de la media como de la incertidumbre. Los resultados revelan que la incertidumbre del precio internacional del petróleo no tiene un impacto inmediato en los rendimientos del mercado accionario. No obstante, los resultados muestran la presencia de efectos asimétricos de corto plazo ante choques negativos y positivos del precio internacional del petróleo.

\section{Códigos JEL: C32, G10, G15, Q43}

Palabras clave: Precios del Petróleo; Rendimientos Accionarios; Volatilidad; Modelos GARCH; Energía; Mercados Emergentes

*Autor para correspondencia.

Correo electrónico: dorobe@correo.azc.uam.mx (D. Rodríguez Benavides)

La revisión por pares es responsabilidad de la Universidad Nacional Autónoma de México. 


\begin{abstract}
In this work, the impact of oil price uncertainty on Mexican stock market returns is examined. The uncertainty of the international oil price is approximated through the conditional standard deviation of the error forecast one step ahead of the variation in the oil price. To this end, a SVAR-MGARCH-in-mean model was estimated with monthly data of the international oil price returns and the Mexican Stock Exchange price and quotation index, both in real terms from January 1975 to September 2018, with the main advantage being that it allows the simultaneous estimation of both the mean and the uncertainty. The results reveal that the uncertainty of the international oil price does not have an immediate impact on stock market returns. However, the results show the presence of short-term asymmetric effects in the face of negative and positive shocks in the international oil price.
\end{abstract}

JEL Codes: C32, G10, G15, Q43

Keywords: Oil Prices; Stock Returns; Volatility; GARCH Models; Energy; Emerging Markets

\title{
Introducción
}

En fechas recientes, los precios mundiales de los energéticos han mostrado inestabilidad, Aye (2015). Incluso, se han presentado periodos de gran volatilidad en los precios internacionales del petróleo aun cuando su nivel medio ha permanecido constante, Baskaya et al. (2013). La volatilidad es una característica inherente del precio del petróleo en general debido a su amplio uso como insumo en el proceso de producción y como bien de consumo final, Swanepoel (2006). De igual forma, se reconoce que la volatilidad del precio del petróleo puede ejercer una gran influencia en la economía global. Esta naturaleza ha despertado la preocupación de los responsables de la política económica, las instituciones internacionales, los políticos y los inversionistas sobre la posibilidad de un impacto perjudicial en la macroeconomía, Aye (2015). En consecuencia, los investigadores se han interesado cada vez más en comprender la naturaleza del vínculo entre la volatilidad del precio del petróleo y el desempeño macroeconómico.

Diversos estudios se han enfocado en el impacto de la incertidumbre de los precios del petróleo en algunas variables macroeconómicas reales tales como el consumo, la inversión y el crecimiento. A la par de este interés por el estudio del impacto de la volatilidad y la incertidumbre de los precios del petróleo en la actividad económica, la reciente crisis financiera mundial también ha dirigido la atención al mercado de valores. En este contexto, entender cómo impactan los precios del petróleo a las acciones se ha vuelto relevante y crítico para distintos fondos como son los de coberturas, de índices de materias primas y para otras instituciones financieras, Maghyereh y Awartani (2016).

Teóricamente, los precios de los activos se determinan por los flujos de efectivo descontados, Fisher (1930) y Williams (1938). De esta manera, los factores que inciden en los flujos de efectivo esperados tienen un impacto significativo en los precios de las acciones, Bass (2017). En términos generales, cualquier aumento en el precio del petróleo resultaría en un aumento de los costos, restringiendo las ganancias y en mayor medida, causaría una disminución en el valor de los accionistas. Por lo tanto, cualquier incremento en el precio del petróleo debería ir acompañado de una disminución en los precios de las acciones (Filis et al., 2011).

De acuerdo con Bjornland (2009) y Jiménez-Rodríguez y Sánchez (2005), en un país exportador de petróleo un incremento en el precio del petróleo tiene un impacto positivo en virtud de que este incrementa el ingreso de dicho país. Si el ingreso agregado se incrementa, el 
gasto y la inversión aumentan, lo cual a su vez eleva la productividad y reduce el desempleo. Bajo esta situación, el mercado accionario tiende a responder positivamente.

Mientras que en un país importador de petróleo un incremento del precio del petróleo puede traer consigo efectos adversos, LeBlanc y Chinn (2004) y Hooker (2002), ya que el petróleo es uno de los principales factores de producción utilizados por las empresas y los hogares. Esto lleva a un aumento en los costos, Filis et al. (2011), Arouri y Nguyen (2010), Backus y Crucini (2000) y Kim y Loungani (1992). Este aumento de precios se transfiere a empresas y hogares, lo que puede propiciar presiones inflacionarias y caída de la demanda de las familias con una función de demanda elástica, Hamilton (1988). La reducción de la demanda y el aumento de las expectativas de inflación conducen a una disminución de la producción y el empleo, Lardic y Mignon (2006), Brown y Yücel (2002) y Davis y Haltiwanger (2001). Deteriorando en última instancia el entorno macroeconómico y propiciando una reacción negativa del mercado de valores, Sadorsky (1999) y Jones y Kaul (1996). Filis et al. (2011) señalan que los choques en los precios del petróleo pueden influir en el mercado de valores a través de la incertidumbre, que a su vez afecta al mercado de valores en función de su procedencia, ya sea del lado de la oferta o de la demanda. Si el choque en el precio del petróleo proviene del lado de la demanda la reacción del mercado de valores puede ser positiva, si por el contrario proviene del lado de la oferta, el impacto puede ser negativo, Bass (2017).

Ross (1989) sugiere que la volatilidad de los cambios de precios puede ser una buena aproximación de la tasa de flujo de información en los mercados financieros. En consecuencia, los choques de volatilidad del precio del petróleo pueden tener un impacto en los rendimientos reales de las acciones.

Otro aspecto interesante que a los investigadores les ha llamado la atención es porque el impacto del precio de petróleo es asimétrico, es decir porque el impacto de un incremento del precio del petróleo no es el mismo que el de una reducción, Aye (2015). Sadorsdy (1999) provee dos posibles explicaciones acerca de los efectos asimétricos de los choques del precio internacional del petróleo en la actividad económica. La primera sugiere que lo importante es la magnitud de los cambios en los precios relativos. En tanto que la segunda tiene que ver con la irreversibilidad de la inversión bajo incertidumbre, y que hace hincapié en que hay un valor de opción asociado con la espera para invertir.

No obstante, a pesar de este creciente interés, la investigación sobre este tema se ha llevado a cabo en su mayoría para los países desarrollados, los estudios sobre países en desarrollo son limitados, la mayoría se centra en los mercados de valores en los países importadores de petróleo y sólo unos pocos se han efectuado para los países exportadores de petróleo (Awartani y Maghyereh, 2013; Basher et al., 2012; Chen, 2010; Elder y Serletis, 2010; Maghyereh y AlKandari, 2007; Maghyereh y Awartani 2016; Jones y Kaul, 1996; Maghyereh, 2004; Kilian y Park, 2009; Masih et al., 2011; Sadorsky, 1999; Wei, 2003).

En este trabajo pretendemos determinar si la incertidumbre asociada con el precio internacional del petróleo incide en el mercado accionario de México. Para tal fin, empleamos un modelo bivariado general modificado que incorpora un término GARCH en una de las ecuaciones de la media, evitando así el problema del regresor generado planteado por Pagan (1984), el cual está relacionado con la estimación de los parámetros de la función de varianza por separado de los parámetros de la media condicional, al estimar simultáneamente todos los parámetros a través del método de máxima verosimilitud con información completa de acuerdo a lo propuesto por Elder (2004) y Elder y Serletis (2010). 


\section{Revisión de los Estudios Empíricos}

En esta sección proveemos una síntesis de los principales estudios que han abordado la relación entre incertidumbre de los precios del petróleo y los precios del mercado accionario, o sus rendimientos, de países importadores y exportadores de petróleo.

Una breve revisión de la literatura que ha abordado esta relación se presenta en el cuadro 1 .

Tabla 1

Resumen de los estudios empíricos relevantes

\begin{tabular}{|c|c|c|c|}
\hline Autor (año) & Variables & Metodología & Resultados \\
\hline $\begin{array}{l}\text { Hammoudeh } \\
\text { y Li (2005). }\end{array}$ & $\begin{array}{l}\text { Futuros del precio del } \\
\text { petróleo a } 3 \text { meses } \\
\text { NYMEX, MSCI, US } \\
\text { Índice del Petróleo Amex, } \\
\text { US NYSE Índice de } \\
\text { Transportación, IPC de } \\
\text { México, Índice de la Bolsa } \\
\text { de Oslo. }\end{array}$ & VECM, APT & $\begin{array}{l}\text { El aumento de los precios del } \\
\text { petróleo es perjudicial para los } \\
\text { mercados mundiales de capital y } \\
\text { ejerce un impacto positivo en las } \\
\text { existencias relacionadas con el } \\
\text { petróleo. }\end{array}$ \\
\hline Agren (2006) & $\begin{array}{l}\text { Mercados de valores de } \\
\text { Japón, Noruega, Suecia, el } \\
\text { Reino Unido y los Estados } \\
\text { Unidos. }\end{array}$ & $\begin{array}{l}\text { Modelo } \\
\text { asimétrico }\end{array}$ & $\begin{array}{l}\text { Evidencia de efectos de derrame } \\
\text { de volatilidad del precio } \\
\text { internacional del petróleo a los } \\
\text { mercados de valores, con } \\
\text { excepción de Suecia; Los } \\
\text { efectos de derrame de } \\
\text { volatilidad son reducidos en } \\
\text { magnitud, aunque } \\
\text { estadisticamente significativos. }\end{array}$ \\
\hline $\begin{array}{l}\text { Sadorsky } \\
\text { (1999) }\end{array}$ & $\begin{array}{l}\text { Riesgo del precio del } \\
\text { petróleo y } 2 \mathrm{I} \text { rendimientos } \\
\text { de las bolsas de mercados } \\
\text { emergentes }\end{array}$ & $\begin{array}{l}\text { Modelo } \\
\text { multifactorial } \\
\text { internacional }\end{array}$ & $\begin{array}{l}\text { Se encuentra evidencia de que el } \\
\text { riesgo del precio del petróleo } \\
\text { tiene un impacto positivo en los } \\
\text { rendimientos accionarios en los } \\
\text { mercados emergentes. }\end{array}$ \\
\hline $\begin{array}{l}\text { Malik y } \\
\text { Ewing (2009) }\end{array}$ & $\begin{array}{l}\text { Precio del petróleo y tres } \\
\text { rendimientos accionarios } \\
\text { sectoriales de los Estados } \\
\text { Unidos (tecnologia, salud } \\
\text { y servicios al consumidor). }\end{array}$ & $\begin{array}{l}\text { Modelos GARCH } \\
\text { bivariados }\end{array}$ & $\begin{array}{l}\text { Evidencia de una relación } \\
\text { negativa y significativa entre los } \\
\text { rendimientos de los indices } \\
\text { sectoriales y la volatilidad del } \\
\text { precio del petróleo. }\end{array}$ \\
\hline $\begin{array}{l}\text { Arouri et al. } \\
(2011)\end{array}$ & $\begin{array}{l}\text { Transmisión de la } \\
\text { volatilidad entre el } \\
\text { petróleo y los mercados de } \\
\text { valores en Europa y los } \\
\text { Estados Unidos a nivel } \\
\text { sectorial. }\end{array}$ & $\begin{array}{l}\text { Modelo } \\
\text { VARGARCH } \\
\text { generalizado }\end{array}$ & $\begin{array}{l}\text { Propagación directa } \\
\text { generalizada de la volatilidad } \\
\text { entre los rendimientos } \\
\text { accionarios sectoriales y los del } \\
\text { petróleo. Efectos cruzados de } \\
\text { volatilidad del petróleo los } \\
\text { mercados de valores de Europa, } \\
\text { mientras que en Estados Unidos } \\
\text { se observan derramas de } \\
\text { volatilidad en ambos sentidos. }\end{array}$ \\
\hline $\begin{array}{l}\text { Lee y Chiou } \\
\text { (2011) }\end{array}$ & $\begin{array}{l}\text { Relación entre el precio } \\
\text { del petróleo WTI y los } \\
\text { rendimientos del S\&P500 }\end{array}$ & $\begin{array}{lr}\text { Modelo } & \text { GARCH } \\
\text { univariado } r \text { con } \\
\text { cambio de régimen. }\end{array}$ & $\begin{array}{l}\text { Encuentran que ante } \\
\text { fluctuaciones significativas en el } \\
\text { precio del petróleo, los cambios } \\
\text { inesperados en el precio del } \\
\text { petróleo propician impactos } \\
\text { negativos en los rendimientos } \\
\text { del S\&P500, este resultado en }\end{array}$ \\
\hline
\end{tabular}




\begin{tabular}{|c|c|c|c|}
\hline & & & $\begin{array}{l}\text { un régimen de fluctuaciones } \\
\text { menores en el precio del } \\
\text { petróleo no se mantiene. }\end{array}$ \\
\hline $\begin{array}{l}\text { Choi y } \\
\text { Hammoudeh } \\
(2010)\end{array}$ & $\begin{array}{l}\text { Precios del petróleo del } \\
\text { Brent y del WTl, precios } \\
\text { de comodities e indice del } \\
\text { S\&P500. }\end{array}$ & $\begin{array}{l}\text { Modelo GARCH- } \\
\text { DCC simétrico }\end{array}$ & $\begin{array}{l}\text { Muestran evidencia de } \\
\text { correlaciones crecientes entre el } \\
\text { los precios del petróleo Brent, } \\
\text { del petróleo WTI, del cobre, del } \\
\text { oro y de la plata, pero las } \\
\text { correlaciones disminuyen con el } \\
\text { indice S\&P500. }\end{array}$ \\
\hline $\begin{array}{l}\text { Filis et al. } \\
\text { (2011) }\end{array}$ & $\begin{array}{l}\text { Precios del mercado de } \\
\text { valores y los precios del } \\
\text { petróleo para los paises } \\
\text { importadores de petróleo } \\
\text { (Estados Unidos, Alemania } \\
\text { y Países Bajos) y los países } \\
\text { exportadores de petróleo } \\
\text { (Canada, México y Brasil) }\end{array}$ & $\begin{array}{l}\text { Correlaciones } \\
\text { variantes en el } \\
\text { tiempo. }\end{array}$ & $\begin{array}{l}\text { Evidencia de que la correlación } \\
\text { variable en el tiempo no difiere } \\
\text { para las economias importadoras } \\
\text { de petróleo y las exportadoras de } \\
\text { petróleo, la correlación aumenta } \\
\text { positivamente (negativamente) } \\
\text { en respuesta a importantes } \\
\text { choques en el precio del petróleo } \\
\text { del lado de la demanda agregada } \\
\text { (demanda precautoria), como } \\
\text { resultado de las fluctuaciones } \\
\text { del ciclo económico mundial } \\
\text { Los resultados de la correlación } \\
\text { desfasada muestran que los } \\
\text { precios del petróleo tienen un } \\
\text { efecto negativo en todos los } \\
\text { mercados bursátiles. }\end{array}$ \\
\hline $\begin{array}{l}\text { Masih et al. } \\
\text { (2011) }\end{array}$ & Corea del Sur & Modelo VEC & $\begin{array}{l}\text { Impacto negativo de la } \\
\text { volatilidad del precio del } \\
\text { petróleo en los rendimientos } \\
\text { reales de las acciones }\end{array}$ \\
\hline Jouini (2013) & $\begin{array}{l}\text { Precio mundial del } \\
\text { petróleo y rendimientos } \\
\text { accionarios sectoriales en } \\
\text { Arabia Saudita. }\end{array}$ & $\begin{array}{l}\text { Modelo } \\
\text { VAR-GARCH }\end{array}$ & $\begin{array}{l}\text { Evidencia de transmisión de } \\
\text { volatilidad entre precio del } \\
\text { petróleo y los rendimientos } \\
\text { sectoriales. }\end{array}$ \\
\hline $\begin{array}{l}\text { Chang et al. } \\
\text { (2009) }\end{array}$ & $\begin{array}{lcr}\text { Rendimientos } & \text { de } & \text { los } \\
\text { futuros del petróleo WTI y } & \\
\text { los } & \text { rendimientos } \\
\text { accionarios } & \text { de } 10 \\
\text { compañias } & \text { petroleras } \\
\text { mundiales. } & & \\
\end{array}$ & $\begin{array}{l}\text { Modelos GARCH } \\
\text { Multivariados }\end{array}$ & $\begin{array}{l}\text { No encuentran evidencia de } \\
\text { derramas de volatilidad. }\end{array}$ \\
\hline $\begin{array}{l}\text { Arouri et al. } \\
(2012)\end{array}$ & $\begin{array}{l}\text { Mercados petroleros y } \\
\text { accionarios de Europa. }\end{array}$ & $\begin{array}{l}\text { Modelo } \quad \text { VAR- } \\
\text { GARCH }\end{array}$ & $\begin{array}{l}\text { Encuentran efectos } \\
\text { significativos de derramas de } \\
\text { volatilidad entre el precio del } \\
\text { petróleo y rendimientos } \\
\text { accionarios sectoriales. }\end{array}$ \\
\hline $\begin{array}{l}\text { Jiranyakul } \\
\text { (2014) }\end{array}$ & $\begin{array}{l}\text { Incertidumbre del precio } \\
\text { del petróleo y rendimientos } \\
\text { accionarios de Tailandia. }\end{array}$ & $\begin{array}{l}\text { Modelo GARCH } \\
\text { bivariado, pruebas } \\
\text { de causalidad de } \\
\text { Granger por pares }\end{array}$ & $\begin{array}{l}\text { Evidencia de que los } \\
\text { movimientos en el precio real } \\
\text { del petróleo no afectan } \\
\text { adversamente el rendimiento } \\
\text { real del mercado de valores, } \\
\text { pero la volatilidad del precio de } \\
\text { las acciones si afecta el } \\
\text { rendimiento real de las acciones. } \\
\text { Encuentran evidencia de } \\
\text { transmisión de volatilidad }\end{array}$ \\
\hline
\end{tabular}




\begin{tabular}{|c|c|c|c|}
\hline & & & $\begin{array}{l}\text { unidireccional positiva que va } \\
\text { del petróleo al mercado de } \\
\text { valores. }\end{array}$ \\
\hline $\begin{array}{l}\text { Olson et al. } \\
(2014)\end{array}$ & $\begin{array}{l}\text { Mercados de energia y de } \\
\text { renta variable del indice de } \\
\text { energía de Goldman Sachs } \\
\text { y el S\&P500. }\end{array}$ & $\begin{array}{l}\text { Modelo BEKK } \\
\text { Multivariado }\end{array}$ & $\begin{array}{l}\text { Los resultados muestran que los } \\
\text { rendimientos bajos de S\&P500 } \\
\text { causan incrementos sustanciales } \\
\text { en la volatilidad del indice de } \\
\text { energia; Sin embargo, } \\
\text { eneuentran una respuesta debil } \\
\text { de la volatilidad del S\&P500 a } \\
\text { los choques de precios de la } \\
\text { energia. }\end{array}$ \\
\hline $\begin{array}{l}\operatorname{Lin}_{(2014)} \text { et al. } \\
\end{array}$ & $\begin{array}{l}\text { Mercado del petróleo y } \\
\text { mercado accionario de } \\
\text { Ghana. }\end{array}$ & $\begin{array}{l}\text { Modelos } \\
\text { VAR-GARCH, } \\
\text { VAR-AGARCH } \\
\text { DCC-GARCH }\end{array}$ & $\begin{array}{l}\text { Evidencia de transmisión de } \\
\text { volatilidad positiva y } \\
\text { significativa e interdependencia } \\
\text { entre el petróleo y los } \\
\text { rendimientos del mercado de } \\
\text { valores. }\end{array}$ \\
\hline $\begin{array}{l}\text { Basher } \quad y \\
\text { Sadorsky } \\
(2006)\end{array}$ & $\begin{array}{l}\text { Precio del petróleo en un } \\
\text { conjunto de } 21 \\
\text { rendimientos de mercados } \\
\text { de valores emergentes. }\end{array}$ & $\begin{array}{l}\text { Análisis de regresión } \\
\text { agrupado. }\end{array}$ & $\begin{array}{l}\text { Se encuentra un fuerte impacto } \\
\text { estadisticamente significativo. }\end{array}$ \\
\hline $\begin{array}{l}\text { Gupta } \quad y \\
\text { Modise } \\
(2013)\end{array}$ & $\begin{array}{l}\text { Precio del petróleo y } \\
\text { rendimientos del mercado } \\
\text { de valores de Sudáfrica }\end{array}$ & VAR estructural & $\begin{array}{l}\text { Para los paises importadores de } \\
\text { petróleo, el rendimiento de las } \\
\text { acciones aumenta como } \\
\text { resultado de un aumento del } \\
\text { precio del petróleo solo cuando } \\
\text { la economia mundial está } \\
\text { aumentando. En caso de } \\
\text { demanda especulativa y choques } \\
\text { de oferta de petróleo, } \\
\text { disminuyen los rendimientos } \\
\text { accionarios. }\end{array}$ \\
\hline $\begin{array}{l}\text { Wang et al. } \\
\text { (2013) }\end{array}$ & 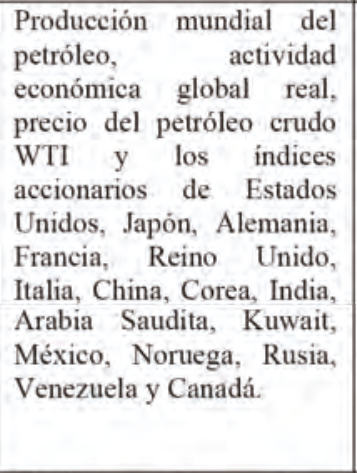 & VAR estructural & $\begin{array}{l}\text { Ninguno de los mercados } \\
\text { bursátiles bajo estudio responde } \\
\text { a los choques del precio del } \\
\text { petróleo. Los mercados de } \\
\text { valores de los paises } \\
\text { exportadores de petróleo } \\
\text { responden positivamente a los } \\
\text { choques de demanda agregada } \\
\text { positivos. Los resultados no son } \\
\text { concluyentes para los choques } \\
\text { de demanda precautorios, para la } \\
\text { mayoría de los mercados de } \\
\text { capitales, los efectos son } \\
\text { insignificantes. }\end{array}$ \\
\hline $\begin{array}{l}\text { Maghyereh y } \\
\text { Awartani } \\
(2016) \text {. }\end{array}$ & $\begin{array}{l}\text { Precios de cierre } \\
\text { semanales de los mercados } \\
\text { de valores de diez paises } \\
\text { incluidos en la muestra y } \\
\text { como proxy del precio del } \\
\text { petróleo emplean el precio } \\
\text { spot del petróleo crudo } \\
\text { Brent. }\end{array}$ & $\begin{array}{l}\text { SVAR-MGARCH } \\
\text { en media. }\end{array}$ & $\begin{array}{l}\text { La incertidumbre del precio del } \\
\text { petróleo es importante en la } \\
\text { determinación de los } \\
\text { rendimientos reales de las } \\
\text { acciones. Existe una relación } \\
\text { negativa y significativa entre la } \\
\text { incertidumbre del precio del } \\
\text { petróleo y el rendimiento real de }\end{array}$ \\
\hline
\end{tabular}




\begin{tabular}{|c|c|c|c|}
\hline & & & $\begin{array}{l}\text { las existencias en todos los } \\
\text { paises de la muestra. La } \\
\text { incidencia del riesgo del precio } \\
\text { del petróleo es más fuerte en } \\
\text { aquellas economias que } \\
\text { dependen en gran medida de los } \\
\text { ingresos del petróleo para crecer. }\end{array}$ \\
\hline Bass (2017) & $\begin{array}{l}\text { Precio internacional del } \\
\text { petróleo y rendimientos del } \\
\text { mercado accionario de } \\
\text { Rusia }\end{array}$ & $\begin{array}{l}\text { SVAR-MGARCH } \\
\text { en media }\end{array}$ & $\begin{array}{l}\text { La incertidumbre de los precios } \\
\text { del petróleo tiene un impacto } \\
\text { positivo y estadísticamente } \\
\text { significativo en los rendimientos } \\
\text { de las acciones. También } \\
\text { encuentra evidencia de efectos } \\
\text { asimétricos en los rendimientos } \\
\text { accionarios ante choques } \\
\text { negativos y positivos de los } \\
\text { precios del petróleo. }\end{array}$ \\
\hline $\begin{array}{l}\text { Kocaarslan et } \\
\text { al. (2017). }\end{array}$ & $\begin{array}{l}\text { Precios de cierre diarios de } \\
\text { los Indices Internacionales } \\
\text { de Capital de Morgan } \\
\text { Stanley, especificamente } \\
\text { de los mercados de } \\
\text { capitales de los BRIC's y } \\
\text { de Estados Unidos asi } \\
\text { como distintos indices de } \\
\text { volatilidad de corto plazo } \\
\text { del Índice S\&P 500, del } \\
\text { tipo de cambio del dólar } \\
\text { contra el euro, del petróleo } \\
\text { y del oro. }\end{array}$ & $\begin{array}{l}\text { Modelos VAR-A- } \\
\text { DCC-EGARCH }(1,1) \\
\text { y VAR-DCC- } \\
\text { EGARCH }(1,1)\end{array}$ & $\begin{array}{l}\text { El impacto de las expectativas } \\
\text { de volatilidad en los mercados } \\
\text { de valores, oro y petróleo de los } \\
\text { Estados Unidos es asimétrico y } \\
\text { dependen del nivel de las } \\
\text { correlaciones. Adicionalmente, } \\
\text { la interdependencia entre los } \\
\text { mercados es impulsada por las } \\
\text { percepciones de riesgo tanto en } \\
\text { los mercados financieros como } \\
\text { en los no financieros. }\end{array}$ \\
\hline $\begin{array}{l}\text { Mensi et al. } \\
\text { (2017). }\end{array}$ & $\begin{array}{l}\text { Precios spot de cierre } \\
\text { diarios del WTI y los } \\
\text { índices de cuatro mercados } \\
\text { de capitales desarrollados } \\
\text { (S\&P 500, STOXX 600, } \\
\text { DJPS y TSX). }\end{array}$ & $\begin{array}{l}\text { Método del modo de } \\
\text { descomposición } \\
\text { variacional (VMD) y } \\
\text { funciones cópula } \\
\text { simétricas y } \\
\text { asimétricas variantes } \\
\text { en el tiempo. }\end{array}$ & $\begin{array}{l}\text { Muestran la existencia de una } \\
\text { dependencia de colas entre los } \\
\text { rendimientos del petróleo y } \\
\text { todos los mercados de valores. } \\
\text { De igual forma, revelan la } \\
\text { existencia de una dependencia } \\
\text { promedio entre los mercados } \\
\text { considerados para los horizontes } \\
\text { a corto plazo. También } \\
\text { encuentran dependencia de colas } \\
\text { a largo plazo entre los mercados } \\
\text { de petróleo y de valores, con } \\
\text { excepción del indice S\&P500. } \\
\text { Adicionalmente, encuentran } \\
\text { fuerte evidencia de altibajos en } \\
\text { las derramas de riesgo } \\
\text { asimétricos del petróleo a los } \\
\text { mercados de valores y viceversa } \\
\text { en diferentes horizontes de } \\
\text { tiempo. }\end{array}$ \\
\hline $\begin{array}{l}\text { Li y } \quad \text { Wei } \\
(2018) .\end{array}$ & $\begin{array}{l}\text { Precio spot diario del } \\
\text { crudo Brent, expresado en } \\
\text { yuanes, y el indice } \\
\text { accionario de la Bolsa de } \\
\text { Shanghai (SSEC). }\end{array}$ & $\begin{array}{l}\text { Método de cópulas } \\
\text { basado en el modo } \\
\text { de descomposición } \\
\text { variacional. }\end{array}$ & $\begin{array}{l}\text { La reciente crisis financiera } \\
\text { aumenta la dependencia entre el } \\
\text { mercado internacional del } \\
\text { petróleo y el mercado accionario } \\
\text { de China, la dependencia a largo } \\
\text { plazo aumenta de manera más }\end{array}$ \\
\hline
\end{tabular}




\begin{tabular}{|c|c|c|c|}
\hline & & & $\begin{array}{l}\text { significativa que la de corto } \\
\text { plazo. } \\
\text { EI VaR del mercado accionario } \\
\text { de China se incrementa } \\
\text { fuertemente alrededor de la } \\
\text { crisis financiera, pero este se } \\
\text { reduce en comparación al riesgo } \\
\text { previo a la crisis. Las derramas } \\
\text { de riesgo del precio del petróleo } \\
\text { al mercado accionario de China } \\
\text { despliegan fuertes caracteristicas } \\
\text { asimétricas. }\end{array}$ \\
\hline $\begin{array}{l}\text { Shahzad ef } \\
\text { al. (2018). }\end{array}$ & 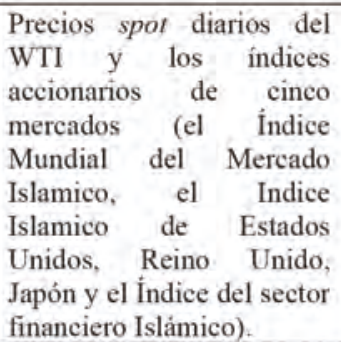 & $\begin{array}{l}\text { Pruebas de } \\
\text { dependencia extrema } \\
\text { a través de cópulas. }\end{array}$ & $\begin{array}{l}\text { Evidencia de dependencia de } \\
\text { cola inferior que varia en el } \\
\text { tiempo entre los mercados de } \\
\text { valores islámicos y petroleros. }\end{array}$ \\
\hline
\end{tabular}

Fuente: Actualizado a partir del cuadro 1 de Bass (2017).

Dados los resultados presentados en el cuadro 1 es posible destacar lo siguiente: i) la investigación sobre el tema se ha concentrado en los países desarrollados, de aquí la relevancia y la importancia de investigar a las economías emergentes; ii) los estudios existentes se dedican principalmente al análisis de la relación entre los precios del petróleo y el valor de mercado de las acciones. Los estudios sobre el impacto del impacto de la incertidumbre de los precios del petróleo en los mercados bursátiles, son escasos; iii) la evidencia de los resultados de estudios previos es mixta; Bass (2017). De esta forma, algunos investigadores han llegado a la conclusión de una relación existente entre la incertidumbre de los precios del petróleo y los rendimientos del mercado accionario mientras que otros han arribado a la conclusión de la ausencia de dicha relación; iv) la mayoría de los estudios analizan de manera simétrica la reacción del mercado bursátil a los choques positivos y negativos de incertidumbre en los precios del petróleo.

\section{Metodología Econométrica}

Con el fin de determinar si la incertidumbre del precio del petróleo incide en la actividad bursátil de México empleamos el modelo desarrollado por Elder (2004) y utilizado en Elder y Serletis (2010), el cual es un modelo bivariado para los rendimientos del mercado de valores (en precios de mercado) y las fluctuaciones del precio del petróleo. El modelo consiste en un VAR estructural modificado para tener en cuenta la heteroscedasticidad condicional en forma paramétrica de un GARCH bivariado en media. La dinámica del sistema estructural se puede resumir mediante una función lineal de las variables de la muestra y los términos relacionados con la varianza condicional, que se pueden representar de la siguiente manera:

$$
A y_{1}=K+\Gamma_{1} y_{t-1}+\Gamma_{2} y_{t-2}+\quad+\Gamma_{p} y_{t-p}+(\Omega M) \sqrt{H_{t}}+\varepsilon_{t}
$$


Expresión en la cual $\operatorname{dim}(A)=\operatorname{dim} \Gamma_{i}$ y son matrices de orden $p \times p, \sqrt{H_{t}}$ es una matriz diagonal y $\Omega(M)$ es una matriz polinomial en el operador rezago. Mientras que $y_{t}$ es un vector que contiene tasas de crecimiento del precio real del petróleo y del índice del mercado accionario en términos reales (rendimientos), $\varepsilon_{t} \mid \Pi_{t-1}$ iid $\left(0, H_{t}\right)$ representa las perturbaciones estructurales no correlacionadas en el sistema y $\Pi_{t-1}$ es el conjunto de información disponible en el tiempo $t-1$.

El modelo planteado en la ecuación (1) permite que la matriz condicional de desviaciones estándar $\left(\sqrt{H_{t}}\right)$ impacten en la media condicional, lo cual permite verificar la hipótesis del impacto de la incertidumbre de los precios del petróleo en los rendimientos accionarios a través de la significancia de uno de los componentes la matriz $\Omega(M)$, dicho coeficiente se espera que resulte negativo y estadísticamente significativo.

La varianza condicional $H_{t}$ es modelada como un GARCH bivariado, el cual es una versión general de la representación de Engle y Kroner (1995):

$$
h_{t}=C_{v}+\sum_{j=1}^{J} F_{i} \operatorname{vec}\left(\varepsilon_{i-j} \varepsilon_{t-j}\right)+\sum_{i=1}^{I} G_{i} h_{t-i}
$$

Donde $\varepsilon_{t} \quad \sqrt{H_{t}} z_{t} ; z_{t}:$ iid $N(0, I)$ donde $C_{v}$ es una matriz $N^{2} \times 1, F$ y $G$ son matrices $N^{2} \times N^{2}$ and $h_{t}=\operatorname{vec}\left(H_{t}\right)$. Esta especificación no garantiza que $H_{t}$ sea definida positiva. De acuerdo con Elder (2004), cuando se incorpora un supuesto que permite identificar el VAR estructural la función de varianza se simplifica sustancialmente, y es posible expresarla en términos de perturbaciones estructurales. Bajo una correlación simultanea de perturbaciones estructurales igual a cero, la matriz de la varianza condicional $H_{t}$ es diagonal, reduciendo el número de requisitos de los parámetros de la función de varianza. De esta forma, es posible escribir la función de la varianza reducida de la siguiente forma:

$$
\operatorname{diag}\left(H_{t}\right)=C_{v}+\sum_{j=1}^{J} F_{j} \operatorname{diag}\left(\varepsilon_{t-j} \varepsilon_{t-j}\right)+\sum_{i=1}^{I} G_{i} \operatorname{diag}\left(H_{t-1}\right)
$$

Donde, diag es el operador que extrae la diagonal de una matriz cuadrada. El segundo y tercer término de la ecuación (3) representan los términos ARCH y GARCH. Al imponer una restricción adicional de que la varianza condicional de $y_{i, t}$ depende únicamente de sus propios errores pasados y de sus varianzas condicionales, las matrices de parámetros $F_{j}$ y $G_{i}$ son también diagonales. La función de la varianza se estima con $J=I=1$, que es una especificación para un modelo VAR-GARCH $(1,1)$ en media.

El modelo bivariado VAR-GARCH en media está representado por las ecuaciones (1) y (3), que se estiman mediante el método de máxima verosimilitud con información completa para evitar el problema del regresor generado relacionado con la estimación de los parámetros de la función de varianza por separado de los parámetros de la media condicional, Pagan (1984) y Bass (2017).

El procedimiento de estimación consiste en maximizar el logaritmo de verosimilitud con respecto a los parámetros estructurales $A, K, \Gamma_{1}, \Gamma_{2}, \quad, \Gamma_{n}, \Omega, F$ y $G$ donde: 
$l_{1}=-\left(\frac{N}{2}\right) \ln (2 \pi)+\frac{1}{2} \ln |B|^{2}-\frac{1}{2} \ln \left|H_{t}\right|-\frac{1}{2} \ln \left|\varepsilon_{t} H_{t}^{-1}\right|$

De acuerdo con Elder y Serletis (2010), valores pre-muestrales de la matriz de varianza condicional, $H_{0}$, se determinan de acuerdo a su esperanza incondicional y es condicional a los valores de la muestra. Adicionalmente, se imponen las siguientes restricciones para asegurar que $H_{t}$ sea definida positiva y $\varepsilon_{t}$ sea de covarianza estacionaria: i) que $C_{v}$ resulte positiva; ii) que $F$ contenga elementos no negativos; y iii) que los eigenvalores de $(F+G)$ tengan modulo menor a uno, Aye (2015) y Bass (2017). Si se cumplen las condiciones de regularidad estándar, el método de máxima verosimilitud con información completa proporciona estimaciones asintóticamente normales y eficientes.

Al imponer las condiciones de identificación usuales en los vectores autorregresivos, es posible estimar los parámetros libres de $B$ sujetos a la condición de rango. Siguiendo a Aye (2015) y Bass (2017), para cumplir con esta condición imponemos la siguiente restricción: los rendimientos del mercado accionario de México responden a los choques de los precios internacionales reales del petróleo, pero no a la inversa.

De igual forma, es posible probar efectos asimétricos de los choques del precio internacional real del petróleo en los rendimientos del mercado accionario en términos reales. Para tal fin, empleamos las funciones de impulso-respuesta. Las funciones de impulso-respuesta del VARGARCH en media se estiman de acuerdo a la propuesta de Elder (2003). Los intervalos de confianza se determinan de acuerdo con el método de Monte Carlo (Hamilton, 1994). Las respuestas a los choques simulados se derivan a través del método de máxima verosimilitud del modelo. A partir de los valores de los parámetros extraídos al azar de la distribución muestral de los estimadores de máxima verosimilitud, los intervalos de confianza se generan a partir de la simulación de 1000 funciones de impulso-respuesta.

\section{Los datos}

Los rendimientos del precio internacional del petróleo se calcularon a partir del precio del West Texas Intermediate (WTI), el cual se obtuvo de dos fuentes: de enero de 1975 a diciembre de 1985 se tomó de Balcilar, Gupta y Miller (2015) mientras que de enero de 1986 a septiembre de 2018 la serie proviene de la Administración de Información Energética de Estados Unidos $(\text { EIA })^{1}$, para expresarlo en términos reales se deflacto con el índice de precios al consumidor de los Estados Unidos. Mientras que los rendimientos del mercado accionario se obtuvieron del Índice de Precios y Cotizaciones (IPC) expresado en moneda local y en términos reales de la Bolsa Mexicana de Valores (BMV), esta última serie se extrajo de Economatica. De esta manera se cuenta con un total de 525 observaciones de las dos series. Ambas series de rendimientos se obtuvieron de sus primeras diferencias logarítmicas. Se opta por emplear los rendimientos en moneda local para aislar el efecto del tipo de cambio, Bass (2017) efectúa un procedimiento similar para el caso de Rusia, por el contrario Aye (2015) convierte el precio internacional de dólares al rand sudafricano con la finalidad de no ignorar el efecto del tipo de cambio. No obstante, Aye (2015) destaca que sus conclusiones se mantienen, independientemente de si el precio del petróleo se expresa en dólares o en la moneda local de Sudáfrica.

\footnotetext{
${ }^{1}$ https://www.eia.gov/dnav/pet/hist/LeafHandler.ashx? $=$ pet\&s=rwtc \&f=m
} 
La gráfica 1 muestra la evolución del precio real del petróleo y sus rendimientos en el periodo de análisis. Por su parte, la gráfica 2 presenta la evolución del Índice de Precios y Cotizaciones en moneda local y sus rendimientos.

Precio real del West Texas Intermediate

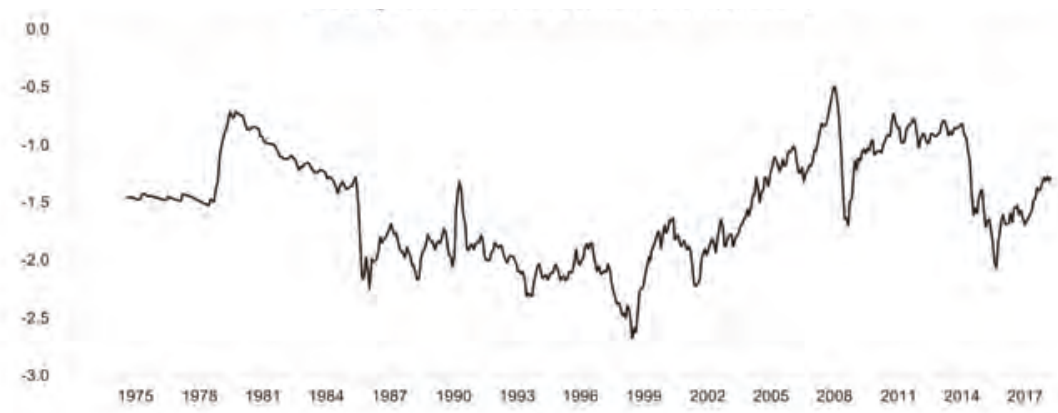

Rendimientos del precio real del West Texas Intermediate.

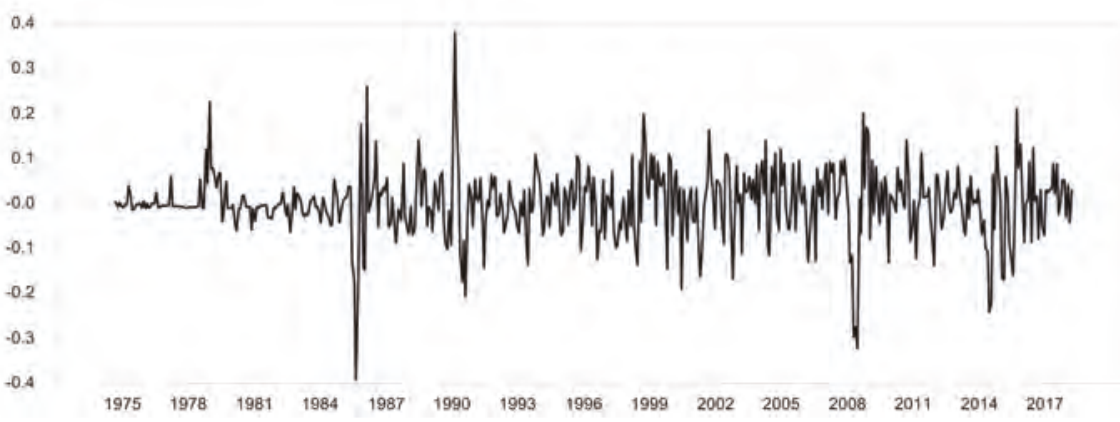

Figura 1. Precio real del petróleo (West Texas Intermediate) y sus rendimientos (1975:1-2018:9).

Rendimientos del precio real del West Texas Intermediate.

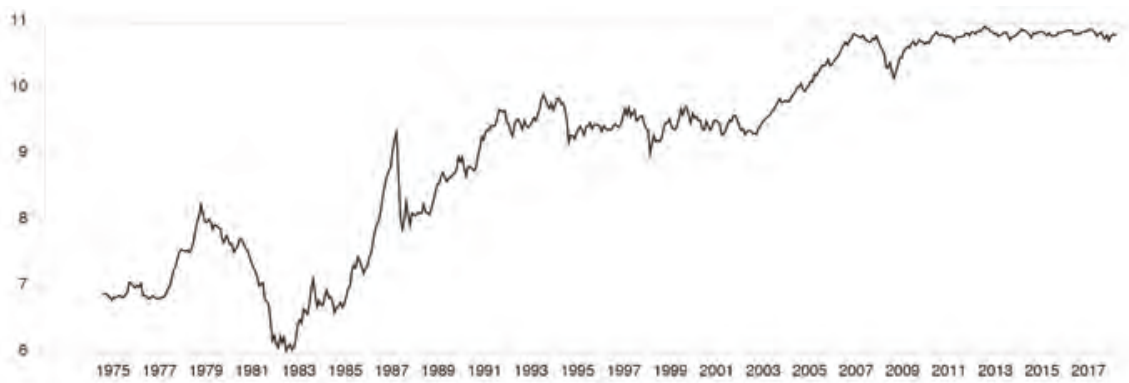




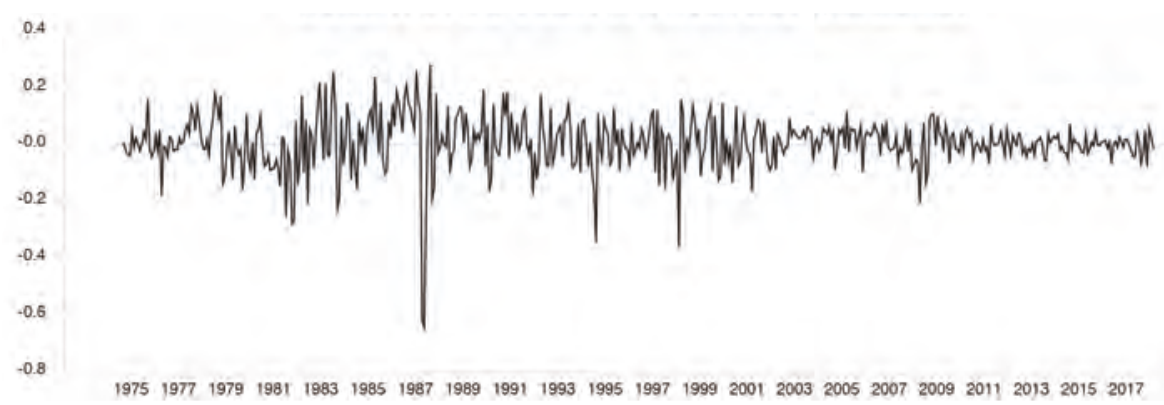

Figura 2. Indice de Precios y Cotizaciones y sus rendimientos en moneda local en términos reales (1975:1-2018:9).

\section{Resultados Empíricos}

En la tabla 2 muestra los resultados de las pruebas de raíz unitaria del multlipicador de Lagrange con dos cambios en el nivel (media).

Tabla 2

Prueba endógena de raíz unitaria del multiplicador de Lagrange con dos rupturas estructurales

Diferencia logarítmica del precio internacional del petróleo en términos reales.

Modelo A: $k=0, T_{B I}=1985: 12, T_{B 2}=2008: 11, N=519$.

Valor critico al 5\%: $-3.842(T=100)$

\begin{tabular}{lcccc}
\hline Parámetro & $\mu_{0}$ & $d_{1}$ & \multicolumn{1}{c}{$d_{2}$} & \multicolumn{1}{c}{$\phi$} \\
\hline Estimado & -0.0134 & -0.1486 & -0.2724 & -0.7178 \\
Estadístico $t$ & -4.0287 & -2.0152 & -3.6494 & -16.9791 \\
\hline
\end{tabular}

Diferencia logarítmica del Índice de Precios y Cotizaciones en términos reales.

Modelo A: $k=0, T_{B 1}=1975: 7, T_{B 2}=2018: 9, N=519$.

Valor critico al 5\%: $-3.842(T=100)$

\begin{tabular}{lcccc}
\hline Parameter & $\mu_{0}$ & $d_{1}$ & $d_{2}$ & \multicolumn{1}{c}{$\phi$} \\
\hline Estimate & -0.0134 & -0.1486 & -0.2724 & -0.7178 \\
$\mathrm{t}$ statistic & -4.0287 & -2.0152 & -3.6494 & -16.9791 \\
\hline
\end{tabular}

Hipótesis nula: $y_{i}=\mu_{0}+d_{1} B_{1 j}+d_{2} B_{z t}+y_{t-1}+v_{1 t}$,

Hipótesis alternativa: $y_{1}=\mu_{1}+\gamma_{t}+d_{1} D_{v i}+d_{2} D_{21}+v_{2 y}$,

donde $D_{j t}=1$ para $t \geq T_{B j}+1, j=1,2$ y 0 de otra forma; $B_{j t}=1$ para $t \geq T_{B j}+1, j=1,2$ y 0 de otra forma; $T_{B j}$

denota la fecha de ruptura.

Cómo se puede ver en el cuadro 2, los resultados de las pruebas de raíces unitarias con dos rupturas en media revelan que ambas series son estacionarias. Teniendo presente que ambas series son estacionarias se estima el modelo SVAR-MGARCH en media con los rendimientos tanto del precios del petróleo en términos reales como con los del Índice de Precios y Cotizaciones también en términos reales con un rezago de acuerdo a lo sugerido por los criterios de Akaike y de Schwarz que coincidieron en este caso. Los resultados de la prueba de especificación se presentan en la tabla 3. 
Tabla 3

Pruebas de Especificación del Modelo1984:2 - 2017:4

\begin{tabular}{lcc}
\hline Modelo VAR y muestra & VAR & VAR con MGARCH-M \\
\hline $\begin{array}{l}\text { Rendimientos del precio real del petróleo } \\
\text { del mercado accionario }\end{array}$ & 7423.46 & 7188.48 \\
\hline
\end{tabular}

Los valores del criterio de Schwarz reportados en el cuadro 3 para los modelos VAR homocedastico y con efectos GARCH en la media, indican que el VAR con efectos GARCH en la media captura mejor las características de los datos en comparación al VAR homocedastico.

El cuadro 4 reporta los coeficientes estimados de las ecuaciones de la varianza condicional para cada una de las variables incorporadas en el modelo. Los valores de los parámetros estimados muestran claramente que los coeficientes de los términos ARCH y GARCH son estadísticamente significativos para ambas variables. Estos resultados proveen soporte adicional a la validez del modelo del modelo SVAR-MGARCH en media.

Tabla 4

Coeficientes Estimados de la Función de Varianza del VAR MGARCH-M

\begin{tabular}{lcccc}
\hline Ecuación de los rendimientos & $\begin{array}{c}\text { Varianza } \\
\text { condicional }\end{array}$ & Constante & $\varepsilon_{i}(t-1)^{2}$ & $H_{i, i}(t-1)$ \\
\hline Del petróleo & $H_{1, l}(t)$ & $\begin{array}{c}32.02^{* *} \\
(12.14)\end{array}$ & $\begin{array}{c}0.456^{* *} \\
(5.49)\end{array}$ & 0.00 \\
& $H_{2,2}(t)$ & $\begin{array}{c}0.473^{* *} \\
(2.25)\end{array}$ & $\begin{array}{c}0.150^{* *} \\
(8.54)\end{array}$ & $\begin{array}{c}0.859^{* *} \\
(55.23)\end{array}$ \\
$\begin{array}{l}\text { Del mercado } \\
\text { accionario }\end{array}$ & & &
\end{tabular}

Notas: Los números entre paréntesis son los estadísticos-t asociados a cada parámetro estimado.

** Denota significancia al nivel del $5 \%$.

* Denota significancia al nivel del 10\%.

El efecto de la incertidumbre del precio internacional real del petróleo en los rendimientos del mercado accionario de México se presenta en la tabla 5. Dicha tabla muestra la estimación puntual del parámetro de interés y su estadístico $t$ asintótico correspondiente entre paréntesis. Como se puede apreciar dicho coeficiente resultó negativo, pero no es significativo. De aquí se puede concluir que para el mercado accionario de México no hay evidencia de que la incertidumbre del precio internacional real del petróleo impacte negativamente en los rendimientos accionarios reales. Este resultado se contrapone a lo encontrado por la mayoría de los estudios efectuados para otros mercados.

Tabla 5

Coeficientes Estimados de la Volatilidad del Petróleo

\begin{tabular}{lc}
\hline & Coeficiente de la volatilidad del petróleo \\
Indicador de la Actividad Económica & $H_{1,1}(t)^{1 / 2}$ \\
\hline IPC & -0.028 \\
& $(-0.29)$ \\
\hline Notas: Los números entre paréntesis son los valores absolutos de los estadísticos t asintóticos. \\
** Denota significancia al nivel del 5\%. \\
* Denota significancia al nivel del 10\%.
\end{tabular}


Por otro lado, el impacto asimetrico de la incertidumbre del precio del petróleo en los rendimientos accionarios se evalúa a través de las funciones de impulso-respuesta, que se simulan a partir de las estimaciones de los parámetros del modelo obtenidos por el método de máxima verosimilitud. Para poder comparar los impulsos con los de un VAR homoscedástico, la magnitud de las respuestas al impulso utilizadas para simular las funciones de impulsorespuesta se basa en un choque del precio del petróleo que es igual a la desviación estándar incondicional del cambio en el precio del petróleo. Para examinar si las respuestas a choques positivos y negativos son simétricas o asimétricas, se simula la respuesta de los rendimientos accionarios a ambos tipos de choques del precio del petróleo. Las funciones de impulso-respuesta (línea negra continua) y las bandas de error a una desviación estándar (líneas punteadas) se presentan en la figura 3. Las funciones de impulso-respuesta muestran que ante un choque positivo inesperado del precio internacional de petroleo propician una reducción instantanea en los rendimientos accionarios del mercado en México, posteriormente tiende a revertirse este efecto y a disiparse después en menos de medio año una vez transcurrido el choque.

Choque positivo del precio del petróleo

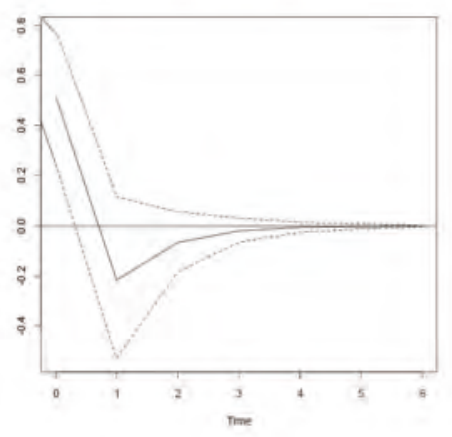

Choque negativo del precio del petróleo

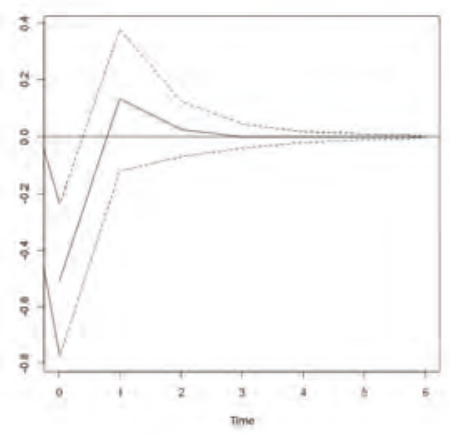

Figura 3. Funciones de Impulso-Respuesta a una desviación estándar a choques positivos y negativos de las variaciones del precio real del petróleo

Por otro lado, las respuestas dinámicas de los rendimientos accionarios a un shock negativo del precio del petróleo tienden a incrementar la rentabilidad del mercado accionario de manera inmediata en el lapso de un mes para después de este tiempo tiende a disiparse este efecto. De esta manera se observa que los rendimientos del mercado accionario muestran efectos asimétricos ante choques positivos y negativos del precio internacional del petróleo. 

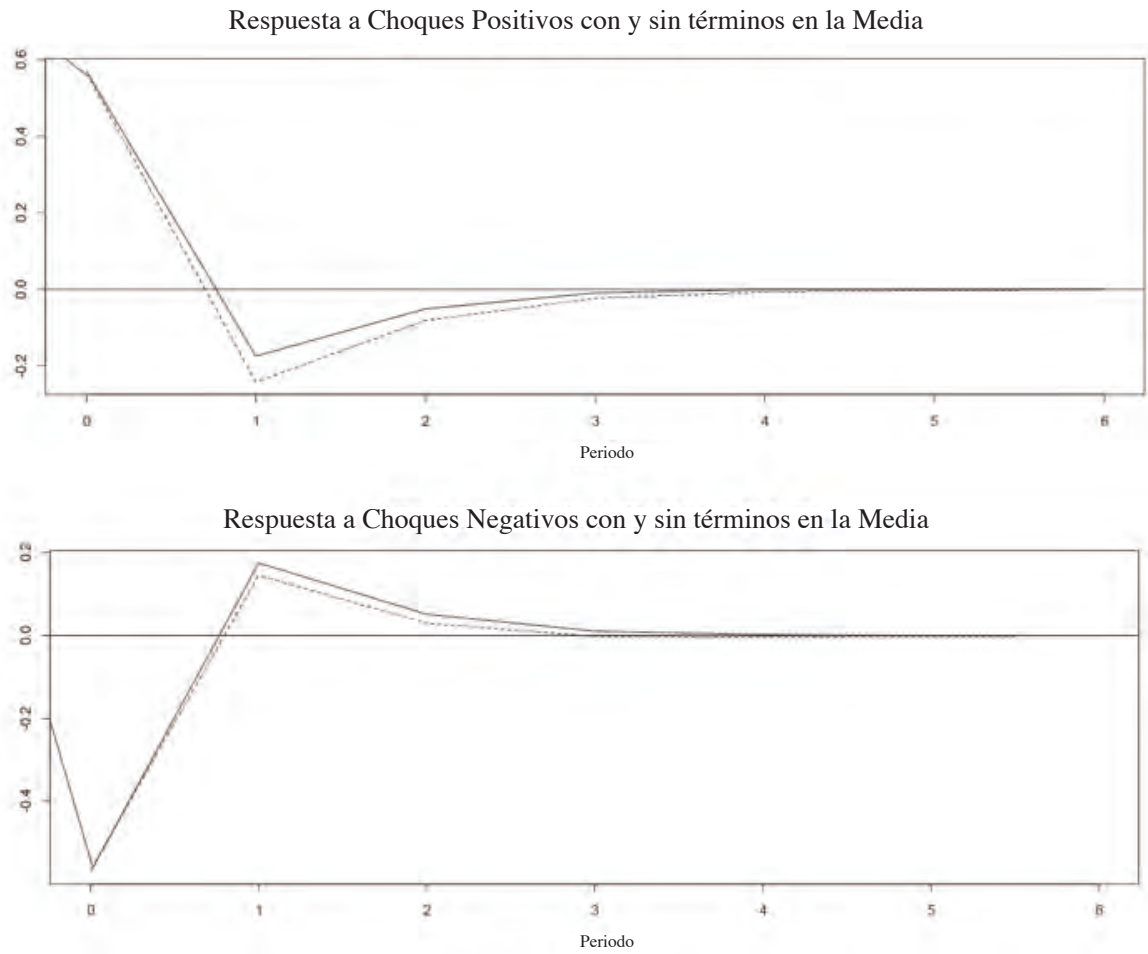

Figura 4. Respuesta a una desviación estándar a choques positivos y negativos del precio real del petróleo con y sin efectos GARCH en media.

Por último, comparamos las respuestas de los rendimientos accionarios a los choques positivos y negativos del precio del petróleo con y sin el término en media como se muestra en la figura 4, donde las bandas de confianza en ambos casos se suprimen para mayor claridad. En la figura 4, las líneas continuas representan la respuesta de los rendimientos accionarios ante un shock en el precio del petróleo en el modelo que incorpora la incertidumbre del precio del petróleo en la ecuación de los rendimientos accionarios, en tanto que las líneas negras representan la respuesta de los rendimientos accionarios después de un choque en el precio del petróleo excluyendo la incertidumbre del precio del petróleo en la ecuación de los rendimientos accionarios. Dicha figura muestra que el patrón asociado con el VAR-GARCH multivariado en media es muy similar a la del VAR homoscedastico. Esto sugiere que el VAR-GARCH multivariado en media es una especificación apropiada del modelo para capturar la influencia de la incertidumbre del precio del petróleo en los rendimientos accionarios.

\section{Conclusiones}

En este trabajo investigamos el efecto de la incertidumbre del precio internacional del petróleo en los rendimientos accionarios del mercado en México a través de un SVARMGARCH en media con datos mensuales en el periodo comprendido entre enero de 1975 a septiembre de 2018. 
La incertidumbre del precio del petróleo se estimó como la desviación estándar condicional del error de pronóstico de un paso adelante del cambio en el precio del petróleo. Con base en el criterio informativo de Akaike, los resultados muestran que el VAR con efectos GARCH en media representan mejor los datos que el VAR homocedásico convencional. De igual forma, no encontramos evidencia que soporte la hipótesis que la incertidumbre del precio internacional del petróleo tiene un impacto negativo inmediato en los rendimientos accionarios en México. Sin embargo, el análisis de los efectos asimétricos a través de las funciones de impulso-respuesta muestra que ante un choque positivo del precio internacional de petroleo los rendimientos accionarios tienden a disminuir de manera abrupta y posteriormente tiende a revertirse este efecto y a disiparse en un lapso de tiempo relativamente corto. Por otro lado, la respuesta dinámica de los rendimientos accionarios a un shock negativo del precio del petróleo es que tienden a incrementarse hasta cerca de un mes una vez transcurrido el choque y después de este lapso de tiempo se revierte este impacto para diluirse después de tres meses.

Lo anterior implica que la respuesta de los rendimientos accionarios a los choques positivos y negativos del precio internacional del petróleo de igual magnitud es asimétrica. La evidencia también muestra que el patrón de las funciones impulso-respuesta de los VAR con y sin efectos en media es muy similar, lo que permite concluir que el modelo VAR con efectos GARCH en media es una especificación apropiada para capturar la influencia de la incertidumbre del precio del petróleo en los rendimientos accionarios.

Es preciso resaltar que nuestro resultado de la no significancia del parámetro que captura el impacto de la incertidumbre asociada con el precio internacional del petróleo en la ecuación de la media de los rendimientos accionarios en México no es consistente con la mayoría de los resultados de estudios empíricos efectuados con esta misma metodología para otros países. No obstante, los resultados de los impactos asimétricos en la dinámica de los rendimientos muestran patrones de comportamiento similar a los encontrados por esos mismos estudios.

Los resultados obtenidos de este estudio tienen posibles implicaciones políticas importantes para los inversionistas, los participantes en el mercado y los formuladores de políticas. Por ejemplo, los inversionistas y los participantes del mercado deben estar conscientes de los vínculos entre la incertidumbre del precio internacional del petróleo y el rendimiento de las acciones cuando utilizan al petróleo para cubrir y diversificar sus portafolios, particularmente en las economías donde el petróleo es importante para el crecimiento económico.

En términos generales, las políticas que reduzcan la volatilidad del precio internacional del petróleo beneficiarán al mercado accionario de México ya que favorecen la reducción de las facturas de importación y la expansión de las exportaciones a través del canal del tipo de cambio, razones por la cual puede no ser exagerada la conveniencia de que el precio del petróleo se mantenga estable.

Una de las principales recomendaciones de política económica que se derivan del presente estudio es que las autoridades gubernamentales pueden coadyuvar en el diseño e implementación de instrumentos y estrategias de cobertura que contribuyan a mitigar el riesgo para los inversionistas en el mercado accionario de México derivado de la incertidumbre asociada al precio internacional de petróleo. No obstante, sería interesante determinar si la incertidumbre del precio internacional del petróleo incide a nivel de acciones en el mercado de capitales. De hecho, en los trabajos sobre el tema se destaca que la incertidumbre del precio del petróleo tiene una mayor incidencia en los mercados de valores de los países exportadores de petróleo que en los de países importadores de petróleo, Maghyereh y Awartani (2016). Esto 
implica que invertir en los países importadores de petróleo puede ser una mejor opción que en los países exportadores de petróleo en términos de diversificación de cartera principalmente en periodos de mayor volatilidad e incertidumbre en el precio internacional del petróleo.

Varias cuestiones quedan pendientes por explorar, por ejemplo el impacto de los choques del precio internacional del petróleo con datos con una mayor frecuencia, ya sea quincenal, semanal o diaria, ya que de acuerdo con Arouri et al. (2012) los datos con frecuencia mensual, pueden ser inadecuados para capturar los mecanismos de transmisión de volatilidad debido a la agregación temporal y a los efectos de compensación.

\section{Referencias}

Agren, M. (2006). Does Oil Price Uncertainty Transmit to Stock Markets? Working Paper, Department of Economics, Uppsala University, No. 2006, 23. Available from: http://www.econstor.eu/bitstream/10419/82778/1/wp2006-023. pdf.

Arouri, M.E.H. y Nguyen, D.K. (2010). Oil prices, stock markets and portfolio investment: Evidence from sector analysis in Europe over the last decade. Energy Policy, 38, 4528-4539. https://doi.org/10.1016/j.enpol.2010.04.007

Arouri, M.E.H., Jouini, J., Ngugen, D.K. (2011). Volatility spillovers between oil prices and stock sector returns: Implications for portfolio management. Journal of International Money and Finance, 30(7), 1387-1405. https://doi. org/10.1016/j.jimonfin.2011.07.008

Arouri, M.E.H., Jouini, J., Ngugen, D.K. (2012). On the impacts of oil price fluctuations on European equity markets: Volatility spillover and hedging effectiveness. Energy Economics, 34, 611-617. https://doi.org/10.1016/j. eneco.2011.08.009

Aye, G. (2015). Does oil price uncertainty matter for stock returns in South Africa?. Investment Management and Financial Innovations, 12(1):179-188.

Awartani, B. y Maghyereh, A. (2013). Dynamic spillovers between oil and stock markets in the Gulf Cooperation Council Countries. Energy Economics, Vol. 36 No. 2, pp. 28-42. https://doi.org/10.1016/j.eneco.2012.11.024

Balcilar, M., Gupta, R. and Miller, S.M. (2015). Regime switching model of US crude oil and stock market prices: 1859 to 2013. Energy Economics, doi: 10.1016/j.eneco.2015.01.026

Basher, S.A., Sadorsky, P. (2006). Oil price risk and emerging stock markets. Global Finance Journal, 17, 224-251. https://doi.org/10.1016/j.gfj.2006.04.001

Basher, S.A., Haug, A.A. y Sadorsky, P. (2012). Oil prices, exchange rates and emerging stock markets. Energy Economics, Vol. 34 No. 1, pp. 227-240. https://doi.org/10.1016/j.eneco.2011.10.005

Baskaya, Y.S., Hülagü, T. and Küçük, H. (2013). Oil price uncertainty in a small open economy, Central Bank of the Republic of Turkey 2013, Working paper No: 13/09.

Bass, A. (2017). Does oil prices uncertainty affect stock returns in Russia: A bivariate GARCH-in-mean approach. International Journal of Energy Economics and Policy, 7(4), 224-230.

Backus, K.D. and Crucini, M.J. (2000). Oil prices and the terms of trade. Journal of International Economics, 50, pp. 185-213. https://doi.org/10.1016/S0022-1996(98)00064-6

Bjornland, C.H. (2009). Oil price shocks and stock market booms in an oil exporting country. Scottish Journal of Political Economy, 2 (5), pp. 232-254. https://doi.org/10.1111/j.1467-9485.2009.00482.x

Brown, P.A.S. y Yücel, M.K. (2002). Energy prices and aggregate economic activity: An interpretative survey. The Quarterly Review of Economics and Finance, 42, 193-208. https://doi.org/10.1016/S1062-9769(02)00138-2

Chang, C.L., McAleer, M. y Tansuchat, R. (2009). Volatility Spillovers between Crude Oil Futures Returns and Oil Company Stock Returns. Working Paper CARF-F-157, University of Tokyo.

Chen, S.S. (2010). Do higher oil prices push the stock market into bear territory?. Energy Economics, Vol. 32 No. 2, pp. 490-495. https://doi.org/10.1016/j.eneco.2009.08.018

Choi, K., Hammoudeh, S. (2010). Volatility behavior of oil, industrial commodity and stock markets in a regime-switching environment. Energy Policy, 38, 4388-4399. https://doi.org/10.1016/j.enpol.2010.03.067 
Davis, J.S. y Haltiwanger, J. (2001). Sectoral job creation and destruction responses to oil price changes. Journal of Monetary Economics, 48, 465-512. https://doi.org/10.1016/S0304-3932(01)00086-1

Elder, J. (2003). An impulse response function for a vector auto regression with multivariate GARCH-in-mean. Economic Letters, 79, 21-26. https://doi.org/10.1016/S0165-1765(02)00283-5

Elder J. (2004). Another perspective on the effects of inflation uncertainty. Journal of Money, Credit and Banking, 36(5), 911-28.

Elder J, Serletis A. (2009). Oil price uncertainty in Canada. Energy Economics, 31(6), 852-856. https://doi. org/10.1016/j.eneco.2009.05.014

Elder, J., y Serletis, A. (2010). Oil price uncertainty. Journal of Money, Credit and Bank. 42(6), 1137-1159. https://doi. org/10.1111/j.1538-4616.2010.00323.x

Elder J. y Serletis A. (2011). Volatility in oil prices and manufacturing activity: an investigation of real options. Macroeconomic Dynamics, 15(Supplement 3), 379-395. https://doi.org/10.1017/S1365100511000630

Filis, G., Degiannakis, S., y Floros, C. (2011). Dynamic correlation between stock market and oil prices: The case of oil-importing and oil-exporting countries. International Review of Financial Analysis, 20, 152-164. https://doi. $\operatorname{org} / 10.1016 /$ j.irfa.2011.02.014

Fisher, I. (1930). The Theory of Interest. New York: Macmillan.

Gupta, R. y Modise, M.P. (2013). Does the source of oil price shocks matter for South African stock returns? A structural VAR approach. Energy Economics, 40(1), 825-831. https://doi.org/10.1016/j.eneco.2013.10.005

Hamilton, D.J. (1988). A neoclassical model of unemployment and the business cycle. Journal of Political Economy, 96, 593-617.

Hamilton, J.D. (1994). Time Series Analysis. Princeton, NJ: Princeton University Press.

Hammoudeh, S. and H. Li (2005). Oil sensitivity and systematic risk in oil-sensitive stock indices. Journal of Economics and Business 57(1): 1-21. https://doi.org/10.1016/j.jeconbus.2004.08.002.

Hooker, A.M. (2002). Are oil shocks inflationary? Asymmetric and nonlinear specifications versus changes in regime. Journal of Money, Credit and Banking, 34 (2), pp. 540-561.

Jimenez-Rodriguez, R., Sanchez, M. (2005). Oil price shocks and real GDP growth: Empirical evidence for some OECD countries. Applied Economics, 37(2), 201-228. https://doi.org/10.1080/0003684042000281561

Jiranyakul, K. (2014). Does Oil Price Uncertainty Transmit to the Thai Stock Market? MPRA Paper No. 56527. Available from: http://www.mpra.ub.uni-muenchen.de/56527. http://dx.doi.org/10.2139/ssrn.2450974

Jones, C.M. y Kaul, G. (1996). Oil and the stock markets. The Journal of Finance. Vol. 51 No. 2, pp. 463-491. https:// doi.org/10.1111/j.1540-6261.1996.tb02691.x

Jouini, J. (2013). Return and volatility interaction between oil prices and stock markets in Saudi Arabia. Journal of Policy Modeling, 35 (6), pp. 1124-1144.

Kim, I.M. and Loungani, P. (1992). The role of energy in real business cycle models. Journal of Monetary Economics, 29, pp. 173-189. https://doi.org/10.1016/0304-3932(92)90011-P

Kocaarslan, B., Sari, R., Gormus, A., \& Soytas, U. (2017). Dynamic correlations between BRIC and US stock markets: The asymmetric impact of volatility expectations in oil, gold and financial markets. Journal of Commodity Markets, 7, 41-56. https://doi.org/10.1016/j.jcomm.2017.08.001

Kilian, L. y Park, C. (2009). The Impact of Oil Price Shocks on the U.S. Stock Market. International Economic Review, 50(4), 1267-1278. https://doi.org/10.1111/j.1468-2354.2009.00568.x

Lardic, S. y Mignon, V. (2006). Oil prices and economic activity: An asymmetric cointegration approach. Energy Economics, 34, 3910-3915. https://doi.org/10.1016/j.eneco.2006.10.010

LeBlanc, M. and Chinn, D.M. (2004). Do high oil prices presage inflation? The evidence from G5 countries. Business Economics, 34, pp. 38-48. http://dx.doi.org/10.2139/ssrn.509262

Lee, Y. H. y Chiou, J. S. (2011). Oil sensitivity and its asymmetric impact on the stock market. Energy, 36, 168-174. https://doi.org/10.1016/j.energy.2010.10.057

Li, X., y Wei, Y. (2018). The dependence and risk spillover between crude oil market and China stock market: New evidence from a variational mode decomposition-based copula method. Energy Economics, 74, 565-581. https:// doi.org/10.1016/j.eneco.2018.07.011 
Lin, B., Wesseh, P.K.Jr., Appiah, M.O. (2014). Oil price fluctuation, volatility spillover and the Ghanaian equity market: Implication for portfolio management and hedging effectiveness. Energy Economics, 42, 172-182. https://doi. org/10.1016/j.eneco.2013.12.017

Maghyereh, A. (2004). Oil Price Shocks and Emerging Stock Markets: A Generalized VAR Approach. International Journal of Applied Econometrics and Quantitative Studies, 1, issue 2, p. 27-40. https://doi. org/10.1057/9780230599338_5

Maghyereh, A. y Al-Kandari, A. (2007). Oil prices and stock markets in GCC countries: new evidence from nonlinear cointegration analysis.ManagerialFinance, Vol.33 No.7,pp.449-460.https://doi.org/10.1108/03074350710753735

Maghyereh, A. y Awartani, B. (2016). Oil price uncertainty and equity returns: evidence from oil importing and exporting countries in the MENA region. Journal of Financial Economic Policy. Vol. 8 No. 1, pp. 64-79. https://doi. org/10.1108/JFEP-06-2015-0035

Malik, F. y Ewing, B. (2009). Volatility transmission between oil prices and equity sector returns. International Review of Financial Analysis, 18(3), 95-100. 10.1016/j.irfa.2009.03.003

Masih, R., Peters, S., de Mello, L. (2011). Oil price volatility and stock price fluctuations in an emerging market: Evidence from South Korea. Energy Economics, 33(5), 975-986. https://doi.org/10.1016/j.eneco.2011.03.015

Mensi, W., Hammoudeh, S., Shahzad, S.J.H., Shahbaz, M. (2017). Modeling systemic risk and dependence structure between oil and stock markets using a variational mode decomposition-based copula method. Journal of Banking and Finance, 75, 258-279. https://doi.org/10.1016/j.jbankfin.2016.11.017

Olson, E., Vivian, A.J. y Wohar, M.E. (2014). The relationship between energy and equity markets: Evidence from volatility impulse response functions. Energy Economics, 43, 297-305. https://doi.org/10.1016/j.eneco.2014.01.009

Pagan, A. (1984). Econometric issues in the analysis of regressions with generated regressors. International Economic Review, 25, pp. 221-247.

Ross, S. (1989). Information and volatility: the no-arbitrage Martingale approach to timing and resolution irrelevancy. Journal of Finance, 44, pp. 1-17. https://doi.org/10.1111/j.1540-6261.1989.tb02401.x

Sadorsky, P. (1999). Oil price shocks and stock market activity. Energy Economics, 21, 449-469. https://doi.org/10.1016/ S0140-9883(99)00020-1

Shahzad, S.J.H., Mensi, W., Hammoudeh, S., Rehman, M.U., AI-Yahyaee, K.H. (2018). Extreme dependence and risk spillovers between oil and Islamic stock markets. Emerging Markets Review, 34, 42-63. https://doi.org/10.1016/j. ememar.2017.10.003

Swanepoel, J.A. (2006). The impact of external shocks on South African inflation at different price stages. Journal for Studies in Economics and Econometrics, 30(1), pp. 1-22.

Wang, Y., C. Wu, and L. Yang (2013). Oil price shocks and stock market activities: Evidence from oil-importing and oil-exporting countries. Journal of Comparative Economics 41(4): 1220-1239. https://doi.org/10.1016/j. jce.2012.12.004.

Wei, C. (2003). Energy, the stock market, and the putty-clay investment model. American Economic Review, Vol. 93 No. 1, pp. 311-323. 10.1257/000282803321455313

Williams, B.J. (1938). The theory of investment value. Cambridge: Harvard University Press. 